\title{
Bilateral renal artery stenosis and epidermal nevus syndrome in a child
}

\author{
Fahad Alsohim • Pauline Abou-Jaoude • Jean Ninet • \\ Jean-Pierre Pracros • Alice Phan • Pierre Cochat
}

Received: 14 December 2010 /Revised: 25 May 2011 /Accepted: 31 May 2011 /Published online: 1 July 2011

(C) The Author(s) 2011. This article is published with open access at Springerlink.com

\begin{abstract}
Epidermal nevus syndrome is a rare congenital sporadic neuro-ectodermic disorder, characterized by the presence of epidermal nevi in association with various developmental abnormalities of the skin, eyes, nervous, skeletal, cardiovascular and urogenital systems. We describe a 5-year-old boy with conjunctival lipodermoid, cervical and facial sebaceous nevi who presented at 3 years of age with hypertension due to bilateral renal artery stenosis together with multiple vascular anomalies (aorta, celiac trunk, superior mesenteric artery) as shown by magnetic resonance angiography. Systemic arterial hypertension was difficult to control despite combined anti-hypertensive drugs and the surgical repair of the aortic coarctation.
\end{abstract}

F. Alsohim $(\bowtie) \cdot$ P. Abou-Jaoude $\cdot$ A. Phan

Service de néphrologie, rhumatologie et dermatologie

pédiatriques, Hôpital Femme Mère Enfant,

centre de référence des maladies rénales rares,

Hospices Civils de Lyon,

59 boulevard Pinel,

69500 Bron, France

e-mail: alsohim-md@hotmail.com

J. Ninet

Service de chirurgie cardio-vasculaire, Hôpital Louis Pradel, groupement hospitalier Est,

Bron, France

J.-P. Pracros

Service de radiologie, Hôpital Femme Mère Enfant,

Bron, France

P. Cochat

Université de Lyon,

Lyon, France

F. Alsohim

King Saud university,

Riyadh, Kingdom of Saudi Arabia
Keywords Epidermal nevus syndrome $\cdot$ Hypertension . Aortic stenosis $\cdot$ Renal artery stenosis

\section{Introduction}

Epidermal nevus syndrome is a group of congenital neuroectodermic disorders characterized by epidermal nevi in association with cerebral, ocular, skeletal, and sometimes cardiac and renal abnormalities.

In 1968, Solomon et al. [1] reported the first case of epidermal nevus syndrome. While universal diagnostic criteria have not yet been defined, the diagnosis is usually based on epidermal or sebaceous nevus on the scalp, face, trunk or extremities present at birth or appearing in early childhood, accompanied by various anomalies of the central nervous system, bones, and eyes [2]. Vascular anomalies associated with epidermal nevus syndrome are rare findings, including aneurysm, arteriovenous malformation, vascular coarctation, patent ductus arteriosus, and vascular dysplasia [3, 4]. Only one case of arterial hypertension secondary to renal artery stenosis and narrowing of abdominal aorta in association with epidermal nevus syndrome has been described so far [5]. We report on a 5year-old boy with epidermal nevus syndrome who presented with arterial hypertension due to bilateral renal artery stenosis in association with other severe vascular malformations.

\section{Case report}

A 5-year-old boy with epidermal nevus syndrome without neurological abnormality, diagnosed at birth because of right hemifacial and cervical nevi together with conjuncti- 
val lipodermoid, is reported. The skin biopsy of the cervical nevus at birth found sebaceous nevi, which confirmed the diagnosis of epidermal nevus syndrome. High blood pressure $(140 / 70 \mathrm{mmHg})$ was incidentally discovered at 3 years of age, at the time of surgery for cervical nevi excision. Clinical examination revealed a systolic cardiac ejection murmur of $3 / 6$ at the right upper sternal edge without radiation, weak bilateral femoral pulses, and asymmetrical arterial blood pressure between upper and lower limbs. The neurological examination did not show any deficits or musculoskeletal anomalies.

Echocardiography examination revealed a mild coarctation of the aorta at the isthmus with a nadir pressure gradient of $50 \mathrm{mmHg}$, so that antihypertensive drug therapy (beta-blocker + ACE inhibitor) was initiated. One year later, the echocardiography was repeated because of persistent elevated blood pressure, but no significant change was noted. Magnetic resonance angiography (MRA) showed dilated ascending aorta $(20 \mathrm{~mm}$ in diameter) with a hypoplastic transverse arch $(7 \mathrm{~mm})$ and descending aorta $(10 \mathrm{~mm})$. Surgical repair of the aortic coarctation was carried out at 5 years of age by modified Crafoord technique. Post-surgical echocardiography showed improved blood flow velocity $(3 \mathrm{~m} / \mathrm{s})$ and maximal pressure gradient $(36 \mathrm{mmHg})$ at the aortic isthmus. However, the treatment with beta-blocker and ACE inhibitor could not be discontinued because of ongoing systemic hypertension $(140 / 80 \mathrm{mmHg})$.

Six months later, the patient was referred to our department for further investigation of persistent arterial hypertension $(135 / 82 \mathrm{mmHg})$. There was no clinical evidence of postsurgical recurrent stenosis of the aorta (symmetrical blood pressure in both upper and lower limbs).

There was no sign of impairment of renal function and serum creatinine was within the normal range for age.

Renal Doppler ultrasound revealed normal kidney size with normal differentiation, an accelerated blood flow velocity at the right renal artery ostium and proximal left renal artery $(2 \mathrm{~m} / \mathrm{s})$, at the ostium of the superior mesenteric artery (SMA; $3 \mathrm{~m} / \mathrm{s})$ and as well as the coeliac trunk (4-5 m/s). These findings were confirmed by MRA, which showed multiple stenoses at different levels of the aorta without signs of wall thickness or inflammation (Figs. 1, 2, 3), but with stenosis at the ostium of the left subclavian artery $(5.7 \mathrm{~mm}$, then $10 \mathrm{~mm}$ ), narrowing of the descending thoracic aorta at the isthmus $(8 \mathrm{~mm}$, then $10 \mathrm{~mm})$, proximal stenosis of the coeliac trunk, SMA, right and left renal arteries.

Treatment with beta blocker and ACE inhibitor was continued with a progressive dose increase and close observation of renal function until acceptable control of blood pressure was achieved $(120 / 70 \mathrm{mmHg})$.

At last follow-up, i.e., at 10 years of age, the boy had no overt organ damage with normal growth (height 25 th to 50 th

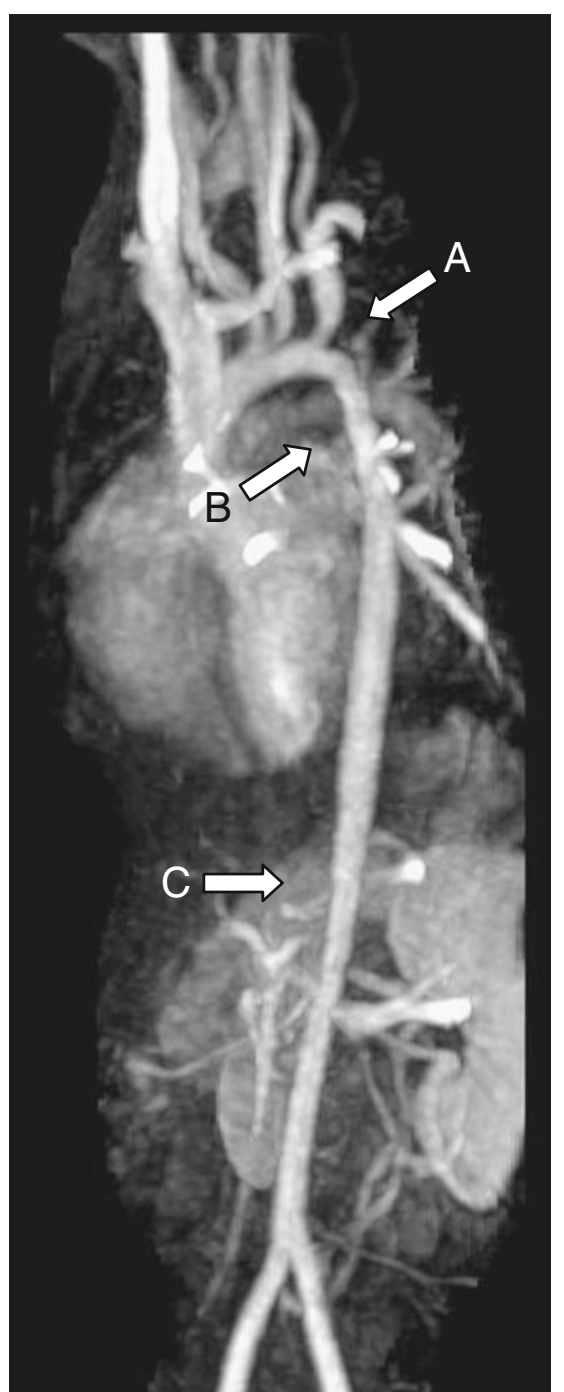

Fig. 1 Left lateral oblique thoracoabdominal aorta-iliac MR angiogram, maximum intensity projection image showing: Arrow A left subclavian artery ostial stenosis, Arrow B the surgical repair site of the coarctation of the thoracic aorta, and Arrow C very mild mid-abdominal aorta narrowing at the level of the coeliac trunk, superior mesenteric artery (SMA), and renal arteries

centile, body weight 75 th to 97 th centile), no remarkable developmental delay, and normal renal function (serum creatinine $38 \mu \mathrm{mol} / \mathrm{l}$, estimated GFR $130 \mathrm{ml} / \mathrm{min} / 1.73 \mathrm{~m}^{2}$ ). Since blood pressure control was currently acceptable under treatment with beta-blocker and ACE inhibitor $(120 / 67 \mathrm{mmHg})$, surgery had been postponed under close surveillance. This strategy was decided according to a surgical team, a radiology team, a nephrology team, and parental input.

\section{Discussion}

Epidermal nevi are circumscribed congenital lesions in the arrangement or quantitative composition of otherwise 


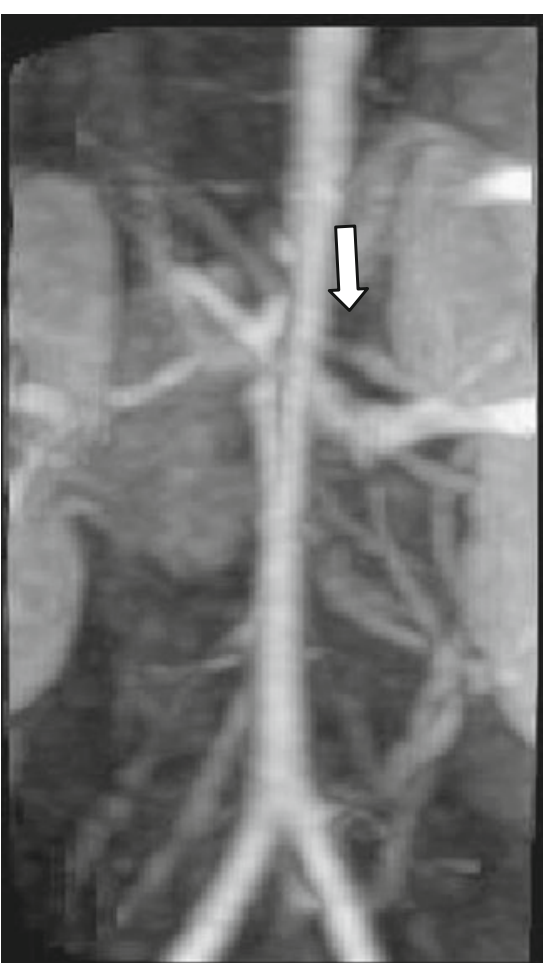

Fig. 2 Frontal view of maximum intensity projection reconstruction image of MR angiogram shows a left renal artery ostial stenosis (arrow)

normal cutaneous elements, and are caused by somatic mutations producing mosaicism [6]. Most often they are linear, following the Blaschko lines, but segmental distribution can be found. The term "epidermal nevus syndrome" refers to sporadic neuro-ectodermic disorders in which epidermal nevi are found in association with deformities and dysplasia of the skin, eyes, brain, skeleton, and heart. Both the cardiovascular and skeletal systems are derived from the mesoderm; thus, epidermal nevus syndrome may result not only from ectodermal, but also from mesodermal defect [7].

Bilateral renal artery stenosis is an unusual vascular malformation associated with epidermal nevus syndrome. In the literature several vascular malformations were reported: coarctation of the aorta, ventricular septal defect, pulmonary artery stenosis, azygos anterior cerebral artery, internal carotid artery occlusion, bilateral vertebral artery occlusion, and unilateral renal artery stenosis $[4,7,8]$; bilateral renal artery stenosis has not been reported so far.

However, such an abnormality has been more commonly reported in other neuro-ectodermic diseases, for example, neurofibromatosis type 1 , in which renal artery stenosis can be detected in $61.5 \%$ of patients [9].

In our patient, the MRA should have been performed earlier to search for another cause of the secondary hypertension in addition to the mild coarctation of the aorta, since blood pressure was not controlled by medical treatment prior to surgery. Although conventional arteriog- raphy could have been more informative than MRA, this might be done later if the situation changes.

As the renin-angiotensin-aldosterone system (RAS) is often activated in patients with renal artery stenosis, our patient had been treated with a regimen including ACE inhibitor and beta-blocker. Although RAS inhibition may induce acute renal failure in some patients with bilateral renal artery stenosis or high-grade stenosis in one kidney, the probability of such a complication appeared to be low, and in most cases, it was reversible with discontinuation of treatment [10].

A percutaneous transluminal angioplasty was not attempted because of known poor results in other neuroectodermic diseases such as neurofibromatosis, and also because of bilateral involvement with a risk of thrombosis and further renal damage compared with acceptable control of blood pressure by medical therapy [11].

The overall therapeutic attitude was made with the motto "primum non nocere" as long as the situation is stable for several years. Advice was gathered from local experts to make the final decision, which was a consensus among nephrologists, vascular surgeons, and radiologists in addition to the approval and support of the parents.

The optimal treatment strategy for renal artery stenosis remains unclear. There are no randomized clinical trials comparing the effects of angioplasty stenting versus medical therapy alone in such patients with vascular dysplasia. In fact this technique had been attempted in a patient with epidermal nevus syndrome and unilateral renal artery stenosis and failed because of the rigidity of the stenotic lesion [5].

According to the guidelines of the Society of Nuclear Medicine and the European Association of Nuclear Medicine, the DMSA scan is not indicated for our patient in the absence of clinical, biological or radiological signs of renal function impairment or deterioration, although it would have been of great value if it had been done earlier as a baseline for renal function evaluation and follow-up.

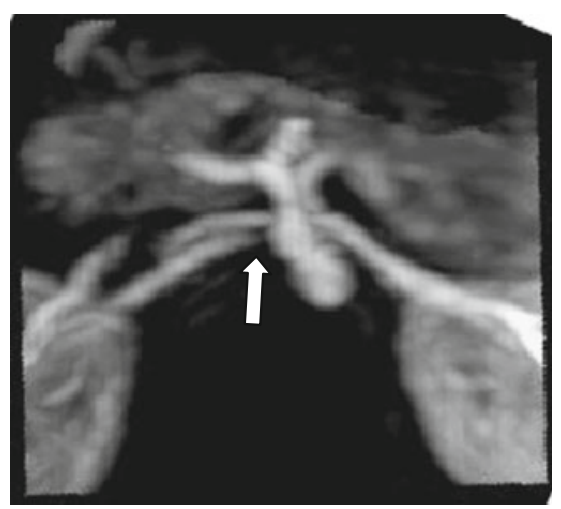

Fig. 3 Axial-oblique view of maximum intensity projection reconstruction image of MR angiogram shows a right renal artery ostial stenosis (arrow) 
In addition to arterial hypertension, the associated diffuse segmental narrowing of the aorta and its branches indicated the association of middle aortic syndrome in this patient, which is a clinical condition generated by segmental narrowing of the descending thoracic and abdominal aorta and their branches, which could be acquired or congenital owing to a developmental anomaly in the fusion and maturation of the paired embryonic dorsal aorta [12].

\section{Conclusion}

Careful screening of renovascular arterial hypertension is strongly recommended in individuals with neuro-ectodermic diseases, especially epidermal nevus syndrome.

Open Access This article is distributed under the terms of the Creative Commons Attribution Noncommercial License which permits any noncommercial use, distribution, and reproduction in any medium, provided the original author(s) and source are credited.

\section{References}

1. Solomon LM, Fretzin DF, Dewald RL (1968) The epidermal nevus syndrome. Arch Dermatol 97:273-285
2. Grebe TA, Rimsza ME, Richter SF, Hansen RC, Hoyme HE (1993) Further delineation of the epidermal nevus syndrome: two cases with new findings and literature review. Am J Med Genet 47:24-30

3. Booth TN, Rollins NK (2002) MR imaging of the spine in epidermal nevus syndrome. AJNR Am J Neuroradiol 23:16071610

4. Rogers M, Mccrossin I, Commens C (1989) Epidermal nevi and the epidermal nevus syndrome: a review of 131 cases. J Am Acad Dermatol 20:476-488

5. Aizawa K, Nakamura T, Ohyama Y, Saito Y, Hoshino J, Kanda T, Sumino H, Nagai R (2000) Renal artery stenosis associated with epidermal nevus syndrome. Nephron 84:67-70

6. Kara B, Inan N, Bayramgurler D, Altintas O, Akbulut A (2008) Epidermal nevus syndrome with azygos anterior cerebral artery. Pediatr Neurol 39:283-285

7. Stavrianeas GN, Kakepis ME (2004) Epidermal nevus syndrome. Orphanet Encyclopedia April 2004, http://www.orpha.net/consor/ cgi-bin/OC_Exp.php?Lng=GB\&Expert=35125

8. Dobyns WB, Garg BP (1991) Vascular abnormalities in epidermal nevus syndrome. Neurology 41:276-278

9. Fossali E, Signorini E, Intermite RC, Casalini E, Lovaria A, Maninetti MM, Rossi LN (2000) Renovascular disease and hypertension in children with neurofibromatosis. Pediatr Nephrol 14:806-810

10. Dworkin LD, Cooper CJ (2009) Renal-artery stenosis. N Eng J Med 361:1972-1978

11. Booth C, Preston C, Clark G, Reidy J (2002) Management of renal vascular disease in neurofibromatosis type 1 and the role of percutaneous transluminal angioplasty. Nephrol Dial Transplant 17:1235-1240

12. Delis KT, Gloviczki P (2005) Middle aortic syndrome: from presentation to contemporary open surgical and endovascular treatment. Perspect Vasc Surg Endovasc Ther 17:187-203 